\title{
CHIRAL AND CONTINUOUS SYMMETRY
}

OF AN $X Y$ SPIN GLASS

\section{ON A TUBE LATTICE}

\author{
M.J. Thill, M. Ney-Niflef and H.J. Hilhorst \\ Laboratoire de Physique Théorique et Hautes Energies 7 \\ Bâtiment 211, Université de Paris-Sud, F-91405 Orsay CEDEx
}

March 30, 2018

LPTHE Orsay 94/23

PACS $05.50+q, 75.10 \mathrm{Nr}$

\footnotetext{
${ }^{\dagger}$ Permanent address: Laboratoire de Physique*, Ecole Normale Supérieure, F-69364 Lyon CEDEx 07
}

* Laboratoire associé au Centre National de la Recherche Scientifique 


\begin{abstract}
We analyse the chiral symmetry in the random $\pm J X Y$ model on a $N \times 2$ square lattice with periodic boundary conditions in the transverse direction. This "tube" lattice may be seen as a twodimensional lattice of which one dimension has been compactified. In the Villain formulation the discrete-valued chiralities or charges associated with the plaquettes of the lattice decouple from the continuous degrees of freedom. The difficulty of the problem lies in the fact that the chiralities interact through the long range "strong" one-dimensional Coulomb potential - which increases linearly with distance - as well as through an exponentially decaying "weak" interaction. By comparing the ground state energies for periodic, antiperiodic, and reflecting boundary conditions in the longitudinal direction, we show that the chiralities and the $X Y$ spins have the same zero- $T$ correlation length exponent, whose exact value $\nu_{c}=0.5564 \ldots$ we determine. The equality of these correlation lengths even in the presence of long range chirality-chirality interactions lends support to the view that chiral glass order cannot be sustained without simultaneous spin glass order.
\end{abstract}




\section{Introduction}

We present a study of the interplay between the spin variables and the chiral variables (chiralities) in the $\pm J X Y$ spin glass. The former correspond to the continuous rotational symmetry of this model, and the latter to its discrete chiral symmetry (i.e., the invariance of the model Hamiltonian under reflection of all the spins with respect to a reference axis), first pointed out by Villain [1, 2, 3]. Below the lower critical dimension, $d_{\ell}$, which is believed to be greater than 2 lcd, the correlation lengths associated with the chiralities and with the spin variables diverge as $T^{-\nu_{c}}$ and $T^{-\nu_{s}}$, respectively, at the zero-temperature $(T)$ critical point. The question of the relation between the two types of variables has become of interest following speculations by Kawamura and Tanemura [5], by Ray and Moore [6] and by Kawamura [7], prompted by Monte Carlo simulations, that below $d_{\ell}$, the two correlation lengths are different, with $\nu_{c}>\nu_{s}$. This suggests that the chiralities will order more easily than the spins in higher dimensions. Consequently, above $d_{\ell}$ there would be a regime of dimensions with long range chiral glass order, but without conventional spin glass order. This possibility receives intuitive support from the idea that discrete symmetry leads to long range order more easily than continuous symmetry does.

Two recent publications [8, 9] address this issue analytically. Both these studies, just like the Monte Carlo work of [5, 6 , consider the finite size scaling of the ground state energy differences between periodic $(\mathrm{P})$, antiperiodic $(\mathrm{AP})$, and reflecting $(\mathrm{R})$ boundary conditions. In one of them, Ney-Nifle and Hilhorst [9] transform the two-dimensional $X Y \pm J$ spin glass on a finite $N \times M$ square lattice into a grand-canonical Coulomb gas problem of which, as is well-known, the logarithmically interacting charges represent the chiral variables. The charges must take half-integer values on the frustrated plaquettes and therefore cannot vanish even in the ground state. In the case of uncorrelated disorder, the plaquettes are randomly and independently frustrated with probability $\frac{1}{2}$, and it is not possible to find the ground state explicitly. For that reason, the subsequent analytic treatment of [9] remains restricted to the example of a rectangular array of frustrated plaquettes with randomly distributed intercolumn distances. In this example, the authors find no evidence for a chiral correlation length diverging faster than the spin correlation length. By a heuristic argument they extend this conclusion to the case of uncorrelated $\pm J$ disorder.

In an earlier investigation, Ney-Nifle et al NHM considered the random $\pm J X Y$ model on a onedimensional ladder lattice, again in the Coulomb gas representation. This problem is exactly solvable, or nearly so, for general disorder, and the conclusions drawn from it are fully coherent with those from the two-dimensional model [9]. However, this model suffers from the drawback that, in the Coulomb gas language, it has only exponentially decaying electrostatic interactions (for reasons explained in that work), so that one may wonder if an essential ingredient of the difficult two-dimensional problem has not been lost.

In the present work, we reconcile the requirements of exact solvability and truly long-range interactions between the chiralities by studying the $\pm J X Y$ spin glass on a $N \times 2$ lattice which is periodic both in the longitudinal and the transverse direction. We work again in the Coulomb gas representation, and apply different boundary conditions. In section 2, we show that, on this two-dimensional lattice with one compactified dimension, the electrostatic interaction decomposes into two components. The first one is a "strong" or charge-charge interaction; it is nothing but the one-dimensional 
Coulomb potential, which increases linearly with distance. The second one is a "weak" interaction: it acts between transversely oriented "dipoles" and decays exponentially with distance. We shall refer to them as the Coulomb and the dipolar interaction, respectively. The Coulomb gas representation of the $X Y$ model Hamiltonian involves, in addition to these two interactions, two supplementary "global" terms that couple the system's total electric dipole moment to the boundary conditions imposed on the Hamiltonian. These extra terms have drawn a certain attention in the recent literature 10, 8, 9, and they play again an important rôle here.

We are not able to solve the ground state problem for the complete Hamiltonian. However, we are able to conclude that in the large $N$ limit, whatever the boundary conditions, the ground state is one of the infinitely many ground states of the "strong" Coulomb interaction combined with one of the global terms. The details of the proof (of largely technical nature) of this fact are given in Appendix A. This set of ground states consists, roughly speaking, of charge configurations in which the long-range Coulomb interaction is screened away as much as possible by the formation of longitudinally oriented dipoles, as exhibited in section 3. The degeneracy within this set is lifted by the weak interaction and by the second global term, which are therefore responsible for the selection of the ground state of the full Hamiltonian and for the energy differences between P, AP, and R boundary conditions. Even though we remain unable to say which member of this set is selected as the true ground state, we are able to describe (in section 4) the domain walls and domain wall energies involved in passing from one boundary condition to another. Using the relation between the correlation lengths and the finite size scaling exponent of the ground state energy differences, we conclude in the final section 5 , for the first time within an $X Y$ spin glass with random $\pm J$ disorder and having a nontrivial long-range interaction between its chiralities, that the spin and the chiral correlation lengths diverge, for $T \rightarrow 0$, with the same exponent $\nu$. We determine its exact value, $\nu=\log _{\frac{8}{3}}^{-1}(3+2 \sqrt{2})=0.5564 \ldots$, in Appendix B.

\section{The tube: a compactified two-dimensional lattice}

In this section we shall exhibit the Hamiltonians of a random $X Y$ model on a $N \times 2$ square lattice with periodic boundary conditions (PBC) in the transverse direction and successively periodic, antiperiodic, and reflecting boundary conditions in the other direction. We call this lattice a tube (see figure 1). It can be viewed as a two-dimensional lattice of which one dimension has been compactified.

First we shall recall the same model on the more general $N \times M$ lattice and then specialise to the tube. The effect of the compactification on the interaction will thereby become clear.

We consider a random $\pm J X Y$ model where the spins are two-component unit vectors whose angles $\phi_{i}$ (with a reference axis) take values in $(-\pi, \pi]$. Two nearest-neighbour spins, $\phi_{i}$ and $\phi_{j}$, have an interaction energy $-J \cos \left(\phi_{i}-\phi_{j}-\pi_{i j}\right)$, where $J$ is a constant, and the $\pi_{i j}$ are quenched random variables that take the values

$$
\pi_{i j}=\left\{\begin{array}{cc}
0, & \text { with probability } \frac{1}{2} \\
\pi, & \text { with probability } \frac{1}{2}
\end{array} .\right.
$$

The partition function is

$$
Z_{X Y}=\int_{-\pi}^{\pi} \prod_{i} d \phi_{i} \mathrm{e}^{\beta J \sum_{<i, j>} \cos \left(\phi_{i}-\phi_{j}-\pi_{i j}\right)} .
$$




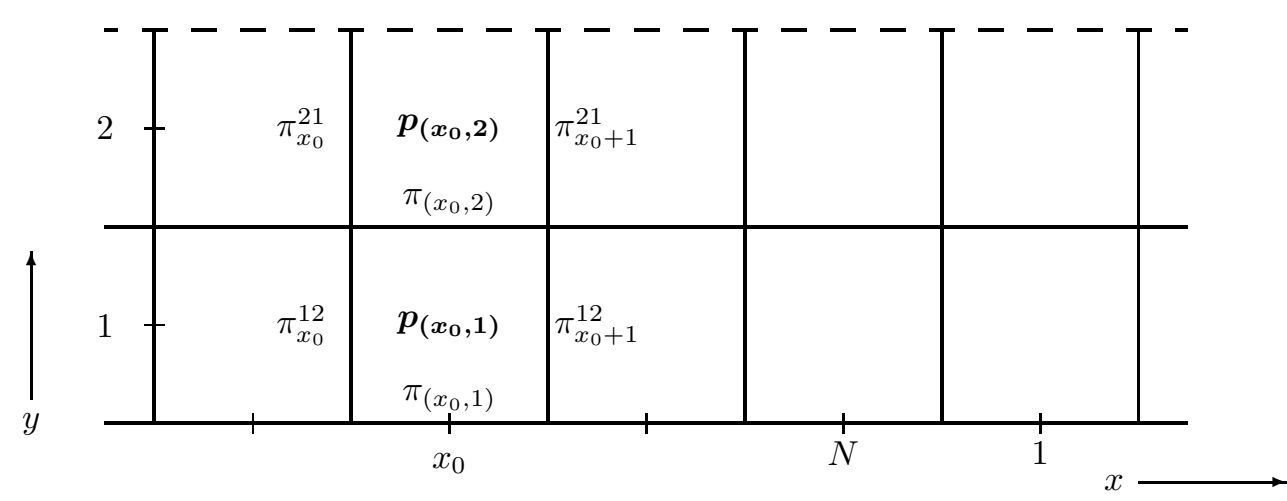

Figure 1: Chiralities on a tube lattice. The bonds on the dashed line are identical with the ones on the lower solid line. The bonds have quenched disorder variables $\pi$ and the plaquette centres frustration variables $p$ associated with them. According to the definition $(2.4)$, one has, for example, $p_{\left(x_{0}, 1\right)}=\left(\pi_{\left(x_{0}, 1\right)}+\pi_{x_{0}+1}^{12}-\pi_{\left(x_{0}, 2\right)}-\pi_{x_{0}}^{12}\right) /(2 \pi)$.

The sum in the exponential in (2.2) runs over all nearest-neighbour bonds of the periodic lattice with the convention that in $\langle i, j\rangle$ the site $j$ is to the right of $i$ (for a horizontal bond) or above $i$ (for a vertical bond). In our notation the site vectors $i=\left(i_{x}, i_{y}\right)$ have half-integer components $i_{x}=\frac{1}{2}, \frac{3}{2}, \ldots, \frac{2 N-1}{2}$ and $i_{y}=\frac{1}{2}, \frac{3}{2}, \ldots, \frac{2 M-1}{2}$.

Since we are interested in the ground state properties of the model, we shall replace (2.2) by the corresponding Villain expression, which is believed to be equivalent to (2.2) in the large- $\beta$ limit [1], 2], and is easier to analyse. The Villain partition function is

$$
Z_{\mathrm{V}}=\int_{-\pi}^{\pi} \prod_{i} d \phi_{i} \sum_{\left\{n_{i j}\right\}} \mathrm{e}^{-\frac{\beta J}{2} \sum_{<i, j>}\left(\phi_{i}-\phi_{j}-\pi_{i j}-2 \pi n_{i j}\right)^{2}},
$$

where the $n_{i j}$ are additional dynamical variables. These $n_{i j}$ are integers and the sum on them ensures that the integrand has period $2 \pi$ in $\phi_{i}-\phi_{j}$. In the following, we set $J=2$.

For each plaquette of the lattice, we define a frustration variable $p_{r}$, with $r=(x, y)$ a vector with integer components $x=1, \ldots, N$ and $y=1, \ldots, M$ that labels the centres of the plaquettes,

$$
p_{r} \equiv \sum_{<i, j>}^{(r)} \epsilon_{i j}^{r} \frac{\pi_{i j}}{2 \pi}
$$

where the sum is restricted to the bonds that define the plaquette $r$. In (2.4), $\epsilon_{i j}^{r}=-1$ or 1 depending on whether one runs through the corners of the triangle $(i j r)$ clockwise or counterclockwise. The frustration variable is integer for nonfrustrated plaquettes and half-integer otherwise.

In (2.3) one can integrate on the continuous degrees of freedom. The algebra (cf. [1, 2, 8, 9]) includes the transformation from the variables $n_{i j}$ to the new discrete variables $q_{r}$ called the "chiralities" of the plaquettes. The chirality $q_{r}$ runs through all integers (half-integers) when $p_{r}$ is integer (half-integer). One shows that the chiralities interact via a Coulomb interaction (which is why they are also called "charges") and that they satisfy the neutrality condition

$$
\sum_{r} q_{r}=0
$$


Recently, Ney-Nifle and Hilhorst [9] (see also 8]) extended the mapping of the $X Y$ Hamiltonian onto a Coulomb gas Hamiltonian by including all the finite size corrections on a $N \times M$ lattice with various boundary conditions. We will now adapt their results to the tube lattice, for which a simplified notation is defined in figure 1 .

\subsection{Periodic boundary conditions}

We shall first consider the $N \times 2$ system with periodic boundary conditions [PBC] in the longitudinal direction. We denote its partition function by $Z_{\mathrm{P}}$. Starting from the more general model $Z_{\mathrm{v}}$ [9], see equation (2.3), we change variables from $n_{i j}$ to the chiralities $q_{r}$ which allows to perform the Gaussian integration on the first set of variables, $\phi_{i}$. Including all numerical prefactors in $Z_{0}^{\mathrm{P}}$, one gets [9]

$$
Z_{\mathrm{P}}=Z_{0}^{\mathrm{P}} \sum_{\left\{q_{r}\right\}} \sum_{n, m} e^{-\beta \mathcal{H}_{\mathrm{P}}} \delta\left(\sum_{r} q_{r}, 0\right),
$$

where $\delta(\cdot, \cdot)$ denotes the Kronecker delta. The additional dynamical variables $n$ and $m$ run over all integers and the $q_{r}$ take integer or half-integer values, as mentioned above. The Hamiltonian $\mathcal{H}_{\mathrm{P}}$, which will be the starting point of our considerations, reads explicitly [9]

$$
\begin{aligned}
\mathcal{H}_{\mathrm{P}}= & \frac{8 \pi^{2}}{N}\left(n+\frac{1}{2} \sum_{x=1}^{N} q_{(x, 1)}+\sum_{x=1}^{N} \frac{\pi_{(x, 1)}}{2 \pi}\right)^{2} \\
+ & 2 \pi^{2} N\left(m+\frac{1}{N} \sum_{x=1}^{N} x\left(q_{(x, 1)}+q_{(x, 2)}\right)+\frac{\pi_{N}^{12}+\pi_{N}^{21}}{2 \pi}\right)^{2} \\
+ & \pi^{2} \sum_{r, r^{\prime}} q_{r} q_{r^{\prime}} U_{N, 2}\left(r-r^{\prime}\right) .
\end{aligned}
$$

We will briefly discuss its meaning. The first two terms are due to the finite system size. They represent a coupling of the horizontal and the vertical component of the total electric dipole moment, respectively, to the quenched disorder. In the third term, $U_{N, M}(R)$ is the interaction between two charges

$$
U_{N, M}(R)=\frac{1}{2 N} \sum_{k_{x}, k_{y}}^{*} \frac{e^{i\left(X k_{x}+Y k_{y}\right)}-1}{\sin ^{2}\left(\frac{k_{x}}{2}\right)+\sin ^{2}\left(\frac{k_{y}}{2}\right)},
$$

with $R=(X, Y), k_{x}=0, \frac{2 \pi}{N}, \ldots, \frac{2 \pi(N-1)}{N}$ and $k_{y}=0, \frac{2 \pi}{M}, \ldots, \frac{2 \pi(M-1)}{M}$. The asterisk indicates that the term $\left(k_{x}, k_{y}\right)=(0,0)$ is left out of the summation.

In $d=2, U_{N, M}(N, M \rightarrow \infty)$ is the two-dimensional Coulomb interaction which varies as a logarithm at large distances [1, 21. For the tube, we will see in what follows that the compactification leads to a decomposition of $U_{N, 2}$ into two parts: a one-dimensional Coulomb interaction that increases linearly with distance and an exponentially decreasing interaction, which is a remnant of a twodimensional dipole-dipole interaction. The appearence of the linear Coulomb interaction and its competition with the dipolar interaction makes the model interesting.

To separate these two interactions in $U_{N, 2}$, we combine the two chiralities of a column $x$ as

$$
\begin{aligned}
q_{x}^{+} & \equiv q_{(x, 1)}+q_{(x, 2)}, \\
q_{x}^{-} & \equiv q_{(x, 1)}-q_{(x, 2)} .
\end{aligned}
$$


Introducing $q_{x}^{+}$and $q_{x}^{-}$in (2.7) and evaluating (2.8) for $N \rightarrow \infty$ in these new variables, we get

$$
\begin{aligned}
\mathcal{H}_{\mathrm{P}}= & \frac{8 \pi^{2}}{N}\left(n+\frac{1}{4} \sum_{x=1}^{N} q_{x}^{-}+\sum_{x=1}^{N} \frac{\pi_{(x, 1)}}{2 \pi}\right)^{2}+\pi^{2} \sum_{x, x^{\prime}=1}^{N} q_{x}^{-} q_{x^{\prime}}^{-} U_{\mathrm{P}}^{-}\left(x-x^{\prime}\right) \\
+ & 2 \pi^{2} N\left(m+\frac{1}{N} \sum_{x=1}^{N} x q_{x}^{+}+\frac{\pi_{N}^{12}+\pi_{N}^{21}}{2 \pi}\right)^{2}+\pi^{2} \sum_{x, x^{\prime}=1}^{N} q_{x}^{+} q_{x^{\prime}}^{+} U_{\mathrm{P}}^{+}\left(x-x^{\prime}\right) .
\end{aligned}
$$

We find that the charges $q_{x}^{+}$interact via the long-range periodised Coulomb potential

$$
\begin{aligned}
U_{\mathrm{P}}^{+}(X) & =\frac{1}{2 N} \sum_{k_{x} \neq 0} \frac{e^{i k_{x} X}-1}{\sin ^{2}\left(\frac{k_{x}}{2}\right)} \\
& \simeq-|X|\left(1-\frac{|X|}{N}\right), \quad N \rightarrow \infty, \frac{|X|}{N} \text { fixed, } 0 \leqq \frac{|X|}{N} \leqq 1 .
\end{aligned}
$$

If $|X|$ is negligible with respect to $N, U_{\mathrm{P}}^{+}(X)$ is the usual one-dimensional Coulomb interaction, linear in $X$. If not, the term $1-\frac{|X|}{N}$ becomes important and reflects the symmetry and periodicity of the lattice.

The charges $q_{x}^{-}$interact via a short-range (dipolar) potential

$$
\begin{aligned}
U_{\mathrm{P}}^{-}\left(x-x^{\prime}\right) & =\frac{1}{2 N} \sum_{k_{x}} \frac{e^{i k_{x}\left(x-x^{\prime}\right)}}{1+\sin ^{2}\left(\frac{k_{x}}{2}\right)} \\
& \simeq \frac{\sqrt{2}}{8}(3-2 \sqrt{2})^{d\left(x, x^{\prime}\right)}, \quad N \rightarrow \infty,\left|x-x^{\prime}\right| \text { fixed },
\end{aligned}
$$

where $d\left(x, x^{\prime}\right)$ is the length of the shortest path between $x$ and $x^{\prime}$, taking into account the periodic geometry. Furthermore, one obtains from the calculation that both potentials have the symmetry properties

$$
\begin{aligned}
& U_{\mathrm{P}}^{ \pm}(X)=U_{\mathrm{P}}^{ \pm}(X+N), \\
& U_{\mathrm{P}}^{ \pm}(X)=U_{\mathrm{P}}^{ \pm}(-X) .
\end{aligned}
$$

Because of the range of the interactions, we will also call the long-range Coulomb interaction between the charges $q_{x}^{+}$strong interaction and the short-range (dipolar) interaction between the charges $q_{x}^{-}$ weak interaction. In the large $N$ limit, for convenience, we rewrite the Coulomb interaction term in (2.10), using (2.11) and charge neutrality, as

$$
\pi^{2} \sum_{x, x^{\prime}=1}^{N} q_{x}^{+} q_{x^{\prime}}^{+} U_{\mathrm{P}}^{+}\left(x-x^{\prime}\right)=-\pi^{2} \sum_{x, x^{\prime}=1}^{N}\left|x-x^{\prime}\right| q_{x}^{+} q_{x^{\prime}}^{+}-\frac{2 \pi^{2}}{N}\left(\sum_{x=1}^{N} x q_{x}^{+}\right)^{2} .
$$

Inserting this expression in (2.10) and writing out also the interaction potentials $U_{\mathrm{P}}^{-}$explicitly, we obtain eventually

$$
\begin{aligned}
\mathcal{H}_{\mathrm{P}}= & \frac{8 \pi^{2}}{N}\left(n+\frac{1}{4} \sum_{x=1}^{N} q_{x}^{-}+\sum_{x=1}^{N} \frac{\pi_{(x, 1)}}{2 \pi}\right)^{2}+\frac{\sqrt{2}}{8} \pi^{2} \sum_{x, x^{\prime}=1}^{N}(3-2 \sqrt{2})^{d\left(x, x^{\prime}\right)} q_{x}^{-} q_{x^{\prime}}^{-} \\
+ & 2 \pi^{2} N\left(m+\frac{\pi_{N}^{12}+\pi_{N}^{21}}{2 \pi}\right)^{2}-4 \pi^{2}\left(m+\frac{\pi_{N}^{12}+\pi_{N}^{21}}{2 \pi}\right) \sum_{x=1}^{N} x q_{x}^{+}-\pi^{2} \sum_{x, x^{\prime}=1}^{N}\left|x-x^{\prime}\right| q_{x}^{+} q_{x^{\prime}}^{+},
\end{aligned}
$$

valid in the large $N$ limit. The task will now be to minimise $\mathcal{H}_{\mathrm{P}}$ with respect to the four variables $q_{x}^{+}$, $q_{x}^{-}, m$, and $n$ in order to find its ground state energy. This will be done in section III. 


\section{$2.2 \quad$ Antiperiodic boundary conditions}

Passing from PBC to antiperiodic boundary conditions (APBC) means changing the sign of the two horizontal bonds that belong to the plaquettes $(N, 1)$ and $(N, 2)$. Under this change frustrated (unfrustrated) plaquettes remain frustrated (unfrustrated). Thus the only modification needed to get the Hamiltonian $\mathcal{H}_{\mathrm{AP}}$ for APBC is to replace $\pi_{(N, 1)}$ by $\pi_{(N, 1)}+\pi$ in the first term in equation (2.15), i.e., to add $\frac{1}{2}$ in the expression between parentheses in that term.

\subsection{Reflecting boundary conditions}

One obtains the Hamiltonian $\mathcal{H}_{\mathrm{R}}$ for the $X Y$ spin glass on an $N \times M$ lattice with reflecting boundary conditions $(\mathrm{RBC})$ in the horizontal direction and $\mathrm{PBC}$ in the vertical direction by replacing the horizontal interactions in one single, but arbitrary column, say $N$, by

$$
\left(\phi_{i}+\phi_{j}-2 \pi n_{i j}-\pi_{i j}\right)^{2} .
$$

This amounts to reflecting the spins on one side of this column with respect to the reference axis. The ensuing modifications in passing to the Coulomb representation result in [9]

$$
Z_{\mathrm{R}}=Z_{0}^{\mathrm{R}} \sum_{\left\{q_{r}\right\}} \sum_{n, m} e^{-\beta \mathcal{H}_{\mathrm{R}}} \delta\left(\left[\sum_{r} q_{r}-\frac{\pi_{N}^{12}+\pi_{N}^{21}}{\pi}\right] \bmod 2,0\right)
$$

with

$$
\mathcal{H}_{\mathrm{R}}=\pi^{2} \sum_{r, r^{\prime}} q_{r} q_{r^{\prime}} U_{\mathrm{R}}\left(r-r^{\prime}\right),
$$

and $r=(x, y)$ labels the centres of the plaquettes on the $N \times M$ lattice as before. We do not recall the explicit, general, form for the potential $U_{\mathrm{R}}$ here, but rather use the variables $q_{x}^{+}$and $q_{x}^{-}$defined in (2.9) and give the explicit expression of $\mathcal{H}_{\mathrm{R}}$ in the case of the tube lattice:

$$
\mathcal{H}_{\mathrm{R}}=\pi^{2} \sum_{x, x^{\prime}=1}^{N} q_{x}^{+} q_{x^{\prime}}^{+} U_{\mathrm{R}}^{+}\left(x-x^{\prime}\right)+\pi^{2} \sum_{x, x^{\prime}=1}^{N} q_{x}^{-} q_{x^{\prime}}^{-} U_{\mathrm{R}}^{-}\left(x-x^{\prime}\right)
$$

where

$$
\begin{aligned}
U_{\mathrm{R}}^{+}(X) & =\frac{N}{2}\left(1-\frac{2|X|}{N}\right), & & N \rightarrow \infty, \frac{|X|}{N} \text { fixed, } 0 \leqq \frac{|X|}{N} \leqq 1, \\
U_{\mathrm{R}}^{-}\left(x-x^{\prime}\right) & =\frac{\sqrt{2}}{8}(3-2 \sqrt{2})^{d\left(x, x^{\prime}\right)} \sigma\left(x, x^{\prime}\right), & & N \rightarrow \infty,\left|x-x^{\prime}\right| \text { fixed },
\end{aligned}
$$

where $\sigma\left(x, x^{\prime}\right)=-1$ if the shortest path between $x$ and $x^{\prime}$ crosses the bond $\pi_{N}^{12}\left(\right.$ or $\left.\pi_{N}^{21}\right)$, and $\sigma\left(x, x^{\prime}\right)=$ 1 otherwise. The interactions $U_{\mathrm{R}}^{+}$and $U_{\mathrm{R}}^{-}$differ furthermore in their symmetry properties from those of $\mathrm{PBC}$ in that one has now

$$
U_{\mathrm{R}}^{ \pm}(X)=-U_{\mathrm{R}}^{ \pm}(X+N),
$$

i.e., antiperiodicity of the interaction potentials. 


\subsection{The Hamiltonians in terms of electric field energy}

In this subsection, we rewrite the strong interaction part of the Hamiltonians (2.15) and (2.19) in terms of an electric field $E_{x}$ :

$$
E_{x}=E_{0}+\sum_{x^{\prime}=1}^{x} q_{x^{\prime}}^{+}
$$

$E_{x}$ is the electric field between $x$ and $x+1$, and $E_{0}$ is a constant background field whose value will be set later in such a way that the volume sum of the energy density $E_{x}$ gives the Coulomb energy of the Hamiltonians. The advantage of this rewriting is clearly seen in Appendix A when properties of the ground states of the Hamiltonians (for large $N$ ) are proven: $E_{x}$ is a local variable, whereas

$\sum_{x^{\prime}=1}^{N} q_{x^{\prime}}^{+} U_{\mathrm{P}, \mathrm{R}}^{+}\left(x-x^{\prime}\right)$ involves all columns of the lattice. To determine the effects on the system's energy when a charge $q_{x_{0}}^{+}$is changed in a given configuration is much easier in terms of the local variable $E_{x_{0}}$, as is manifest in Appendix A.

Squaring (2.22) and summing over $x$, we get

$$
\begin{aligned}
\sum_{x=1}^{N} E_{x}^{2} & =N E_{0}^{2}+2 E_{0} \sum_{x=1}^{N} \sum_{x^{\prime}=1}^{x} q_{x^{\prime}}^{+}+\sum_{x=1}^{N} \sum_{x^{\prime}=1}^{x} \sum_{x^{\prime \prime}=1}^{x} q_{x^{\prime}}^{+} q_{x^{\prime \prime}}^{+} \\
& =N E_{0}^{2}+2 E_{0} \sum_{x=1}^{N}(N-x+1) q_{x}^{+}+\sum_{x^{\prime}=1}^{N} \sum_{x^{\prime \prime}=1}^{N}\left[N+1-\max \left(x^{\prime}, x^{\prime \prime}\right)\right] q_{x^{\prime}}^{+} q_{x^{\prime \prime}}^{+} .
\end{aligned}
$$

Using the identity

$$
\max \left(x^{\prime}, x^{\prime \prime}\right)=\frac{\left|x^{\prime}-x^{\prime \prime}\right|}{2}+\frac{x^{\prime}+x^{\prime \prime}}{2}
$$

and rearranging terms leads to

$$
\begin{aligned}
\sum_{x=1}^{N} E_{x}^{2}= & N\left[E_{0}^{2}+2 E_{0} \sum_{x=1}^{N} q_{x}^{+}+\left(\sum_{x=1}^{N} q_{x}^{+}\right)^{2}\right]-\left(2 E_{0}+\sum_{x=1}^{N} q_{x}^{+}\right) \sum_{x=1}^{N}(x-1) q_{x}^{+} \\
& -\sum_{x^{\prime}=1}^{N} \sum_{x^{\prime \prime}=1}^{N} \frac{\left|x^{\prime}-x^{\prime \prime}\right|}{2} q_{x^{\prime}}^{+} q_{x^{\prime \prime}}^{+} .
\end{aligned}
$$

The value of the constant background field $E_{0}$ is obtained by setting

$$
2 \pi^{2} \sum_{x=1}^{N} E_{x}^{2}=\pi^{2} \sum_{x, x^{\prime}=1}^{N} q_{x}^{+} q_{x^{\prime}}^{+} U_{\mathrm{P}, \mathrm{R}}^{+}\left(x-x^{\prime}\right) .
$$

In the large $N$ limit, this amounts to comparing the expression in 2.25) with the Coulomb interaction part of (2.15) and (2.19) [after insertion of (2.20)]. This gives

$$
E_{0}=\left\{\begin{aligned}
m+\frac{\pi_{N}^{12}+\pi_{N}^{21}}{2 \pi}, & \text { for } \mathcal{H}_{\mathrm{P}} \text { and } \mathcal{H}_{\mathrm{AP}}, \\
-\frac{1}{2} \sum_{x=1}^{N} q_{x}^{+}, & \text {for } \mathcal{H}_{\mathrm{R}},
\end{aligned}\right.
$$

and the Hamiltonians read hence

$$
\begin{aligned}
\mathcal{H}_{\mathrm{P}, \mathrm{AP}}= & 2 \pi^{2} \sum_{x=1}^{N} E_{x}^{2}+\frac{\sqrt{2}}{8} \pi^{2} \sum_{x, x^{\prime}=1}^{N}(3-2 \sqrt{2})^{d\left(x, x^{\prime}\right)} q_{x}^{-} q_{x^{\prime}}^{-} \\
& +\frac{8 \pi^{2}}{N}\left(n+\frac{1}{4} \sum_{x=1}^{N} q_{x}^{-}+\sum_{x=1}^{N} \frac{\pi_{(x, 1)}}{2 \pi}+\frac{1}{2} \delta_{\mathrm{AP}}\right)^{2} \\
\mathcal{H}_{\mathrm{R}}= & 2 \pi^{2} \sum_{x=1}^{N} E_{x}^{2}+\frac{\sqrt{2}}{8} \pi^{2} \sum_{x, x^{\prime}=1}^{N}(3-2 \sqrt{2})^{d\left(x, x^{\prime}\right)} \sigma\left(x, x^{\prime}\right) q_{x}^{-} q_{x^{\prime}}^{-}
\end{aligned}
$$


where

$$
\delta_{\mathrm{AP}}= \begin{cases}0, & \text { for } \mathrm{PBC}, \\ 1, & \text { for } \mathrm{APBC},\end{cases}
$$

with, from (2.6) and (2.17),

$$
\begin{array}{lll}
\sum_{x=1}^{N} q_{x}^{+} & =0 & \text { for } \mathcal{H}_{\mathrm{P}} \text { and } \mathcal{H}_{\mathrm{AP}}, \\
\sum_{x=1}^{N} q_{x}^{+} \bmod 2=\frac{\pi_{N}^{12}+\pi_{N}^{21}}{\pi} \bmod 2 & \text { for } \mathcal{H}_{\mathrm{R}} .
\end{array}
$$

Having established the Hamiltonians for the different boundary conditions, we are now ready to determine those properties of their ground state configurations that are sufficient to calculate the typical energy difference between the ground state energies for $N \rightarrow \infty$.

\section{The ground states for the different boundary conditions}

We summarise the problem to which the preceding sections have led. Each of the three expressions (2.28) should now be minimised with respect to the variables $n,\left\{E_{x}\right\}$, and $\left\{q_{x}^{-}\right\}$. The $\left\{E_{x}\right\}$ are defined in terms of $m$ and $\left\{q_{x}\right\}$ by (2.22) and (2.27), and the $\left\{q_{x}^{+}\right\}$and $\left\{q_{x}^{-}\right\}$are defined in terms of the original charges $\left\{q_{r}\right\}$ by (2.9). The variables $m$ and $n$ are integers, and the $\left\{q_{r}\right\}$ are half-integer or integer according to whether the plaquette $r$ is or is not frustrated.

The ground states of the Hamiltonians (2.28) possess in the large $N$ limit the following properties:

1. The electric field satisfies $\left|E_{x}\right| \leqq \frac{1}{2}$ for $x=1, \ldots, N$. The constant background field takes values $\left|E_{0}\right| \leqq \frac{1}{2}$

2. The charges $q_{(x, 1)}, q_{(x, 2)}$ take the values $0, \pm \frac{1}{2}$.

3. On doubly frustrated columns, the charges $q_{(x, 1)}$ and $q_{(x, 2)}$ are equal if and only if $\left|E_{x-1}\right|=\frac{1}{2}$.

These properties of the ground states, for $N \rightarrow \infty$, are proved in Appendix A. Property 1 indicates that the system's ground state is within the set of states that minimise the electric field (i.e., the Coulomb) energy. For PBC and APBC charge neutrality implies $E_{N}=E_{0}$ (cf. equation (2.22)), whereas for $\mathrm{RBC}$ antiperiodicity of the strong interaction potential leads to $E_{N}=-E_{0}=\frac{1}{2} \sum_{x=1}^{N} q_{x}^{+}$ (from equations (2.22) and (2.27)). Let us just note here that it was conjectured in [2] that, in the ground state of frustrated $X Y$ spin systems, the chiralities (charges) $q_{r}$ are likely to be zero on nonfrustrated plaquettes and take the values $\pm \frac{1}{2}$ on frustrated plaquettes. The above property 2 shows that, for large $N$, this is indeed the case for the tube lattice.

We will now construct the set of states that have properties 1 and 2: We start placing charges $q_{(x, y)}=0, \pm \frac{1}{2}$ successively from $x=1$ to $x=N$, minimising the local electric field energy density 

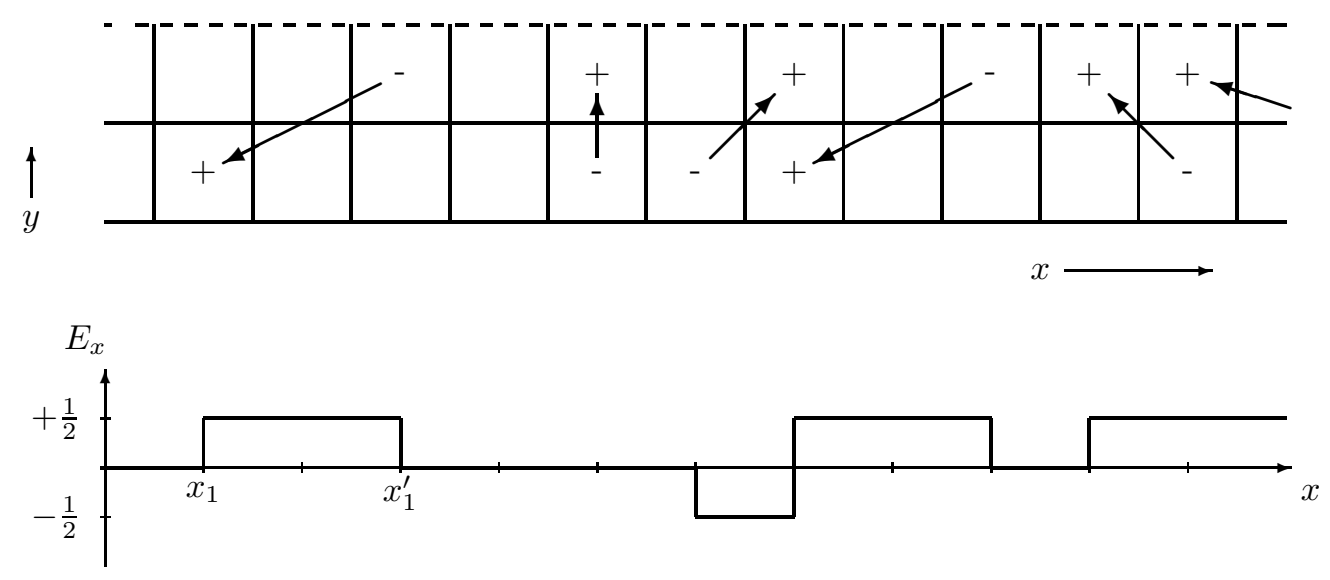

Figure 2: Construction of a state that possesses properties 1 and 2. We start placing charges $q_{(x, y)}$ from the left to the right. We proceed in a way that the local electric field stays as close to 0 as possible. The signs indicate plaquettes with charges $\pm \frac{1}{2}$. Plaquettes with no sign are charge-free (unfrustrated).

$E_{x}^{2}$ at each step and knowing that it changes by a half-integer amount (with $q_{x}^{+}= \pm \frac{1}{2}$ ) on a column with one frustrated plaquette and by an integer amount (with $q_{x}^{+}=0$ for $E_{x-1}=0$ and $q_{x}^{+}=0, \pm 1$ otherwise) on a column with both plaquettes frustrated (see figure 2).

Having thus obtained a state that has properties 1 and 2, we see that it is always possible to partition the nonzero charges $q_{(x, y)}$ into dipoles as in figure 2, grouping together two successive charges of opposite sign along the $x$-axis such that outside the dipoles the electric field is zero. [There is one exceptional case (see figure 3): for $\mathcal{H}_{\mathrm{R}}$ and $E_{0}=\frac{1}{2}$ the last and the first charge placed are not part of any dipole, but of the same sign to take account of the antiperiodicity of the potential $U_{\mathrm{R}}^{+}(X)=-U_{\mathrm{R}}^{+}(X+N)$, respectively $E_{N}=-E_{0}$, as mentioned above.] As one sees from figure 2, e.g. from the dipole containing charges on the columns $x_{1}$ and $x_{1}^{\prime}$, dipole reversals do not change the Coulomb (i.e., electric field) energy of the system. So, the ground state of the system is found within a set consisting of chains of dipoles, satisfying properties 1 and 2 , degenerate in Coulomb energy. The possibility of columns with two identical charges leads to the partition into dipoles not being unique. Property 3, however, introduces a further constraint on the set among which one finds the ground state. Furthermore, one can easily convince oneself that this latter property implies that one can reach every state that satisfies properties 1 to 3 from any other such state, by reversals of dipoles, for any given partition. In particular, the ground state of the system differs, for large $N$ from a state as constructed above (with the additional constraint from property 3 ) by a reversal of dipoles.

The exact ground state configuration remains unknown, but we know that it minimises the Coulomb energy independently of the other energies involved and have characterised the set of Coulomb energy ground states. [Let us just point out here that, when passing from PBC to RBC, one conserves by virtue of $(2.27)$ and $(2.30)$ the property $E_{0}=0$ or $E_{0} \neq 0$ for the ground states at both boundary conditions, due to the fact that $\frac{\pi_{N}^{12}+\pi_{N}^{21}}{2 \pi}$ stays the same [see also Appendix A, especially equation [A.9 ]. So the Coulomb energy stays indeed the same.] The only remaining degrees 

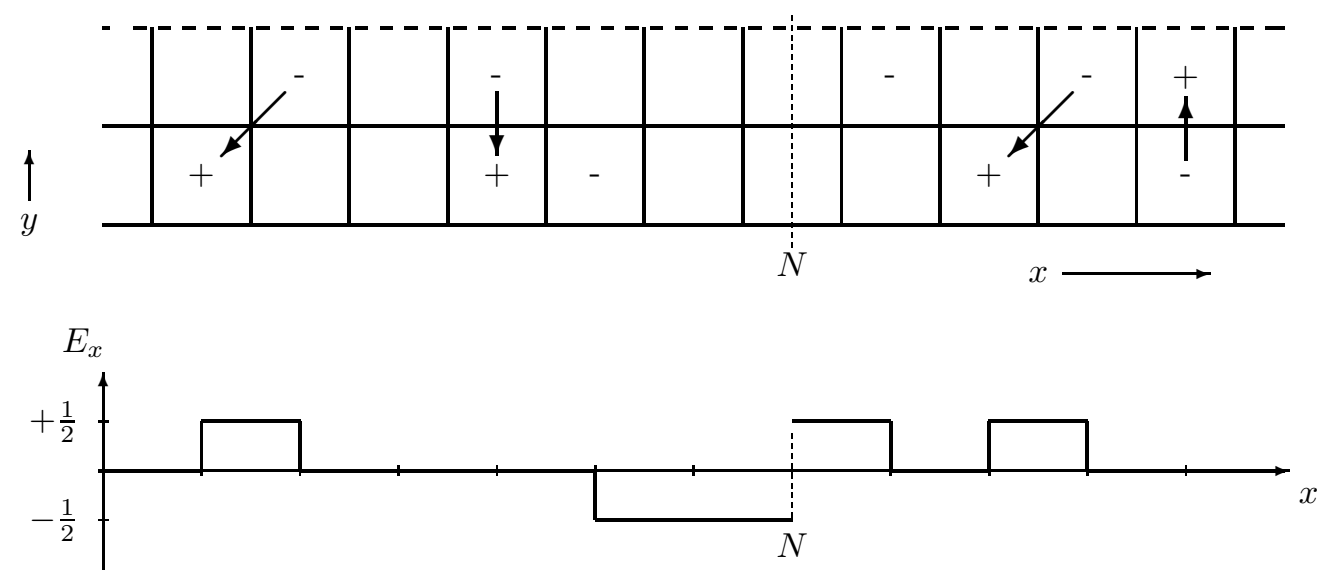

Figure 3: Example of a state with properties 1 and 2 for $\mathrm{RBC}$, while $E_{0}=\frac{1}{2}$. For RBC, proceeding in the construction as described in the text, the last charge to be placed is of the same sign as the first. This is obvious from equation (2.27), $E_{0}=-\frac{1}{2} \sum_{x=1}^{N} q_{x}^{+}$, which indicates that, in general, there is a surplus of two charges $q_{(x, y)}$ with opposite sign to $E_{0}= \pm \frac{1}{2}$ (here $E_{0}=\frac{1}{2}$ ), to take account of the fact that for RBC the potentials $U_{\mathrm{R}}^{ \pm}$are antiperiodic (see 2.21$)$ ). But still all charges, but the first and the last one, can be grouped into dipoles as announced in the text.

of freedom in this set are the directions of single dipoles: The degeneracy is lifted by the other terms in the Hamiltonians of equation (2.28), which fix these directions. The amount of Coulomb energy of the system with the different boundary conditions being the same, it is the effect of these other terms that give rise to the difference between ground state energies when one varies the boundary conditions. We address this issue in detail in the following section. In spite of the fact that we ignore the exact ground state configurations, the above properties s! uffice to analyse and determine th e ground state energy differences for $N \rightarrow \infty$.

\section{Boundary conditions and ground state energy differences}

\subsection{Generalities}

There is a general relation between the finite size scaling of the energy difference, $\Delta E^{(N)} \stackrel{N \rightarrow \infty}{\simeq} J N^{-y}$ (where $J$ is the energy scale), of the ground states of a system for different boundary conditions and the corresponding correlation length, $\xi(T)$, at a finite temperature $T$ : The correlation length $\xi(T)$ is set by $\Delta E^{(\xi)} \sim k_{\mathrm{B}} T$, hence

$$
\xi(T) \sim\left(\frac{k_{\mathrm{B}} T}{J}\right)^{-\frac{1}{y}}, \quad T \rightarrow 0 .
$$

[Let us just note here that the energy difference may be either concentrated in a domain wall or associated with a continuous variation of the order parameter.]

In the Villain model, we may study the spin-spin correlation and the chirality-chirality correlation 
by applying APBC and RBC [5, 8, 9]. So we have to calculate

$$
\begin{array}{ll}
\Delta E_{\mathrm{AP}}^{(N)}=E_{\mathrm{AP}}^{(N)}-E_{\mathrm{P}}^{(N)}, \quad & N \rightarrow \infty, \\
\Delta E_{\mathrm{R}}^{(N)}=E_{\mathrm{R}}^{(N)}-E_{\mathrm{P}}^{(N)}, & N \rightarrow \infty,
\end{array}
$$

where $E_{\mathrm{P}}^{(N)}, E_{\mathrm{AP}}^{(N)}$, and $E_{\mathrm{R}}^{(N)}$ are the ground state energies under P, AP, and R boundary conditions, respectively.

In this section and in Appendix B, we will call a dipole with charges $q_{(x, y)}$ and $q_{\left(x^{\prime}, y^{\prime}\right)}$ slanted, if $y \neq y^{\prime}$, and horizontal, if $y=y^{\prime}$. [With this definition, the slanted ones include the vertical dipoles.] As the probability for a plaquette to be frustrated is the same for all plaquettes, a dipole is as likely to be slanted as horizontal.

Furthermore, writing $U^{-}$for $U_{\mathrm{P}}^{-}$at $\mathrm{PBC}\left(2.12\right.$ ) and for $U_{\mathrm{R}}^{-}$at $\mathrm{RBC}(2.20)$, the weak (dipolar) interaction $U^{-}(\ell)$ has the property

$$
U^{-}(\ell)>\sum_{\ell^{\prime}=\ell+1}^{\infty} U^{-}\left(\ell^{\prime}\right)
$$

for all boundary conditions, so that we may approximate its effect by restricting the interactions of each nonzero charge $q_{x}^{-}$to those with its two nonvanishing neighbouring charges. Upon renumbering the nonzero charges $q_{x}^{-}$on the frustrated columns by a new index $s=1,2, \ldots, N_{c}$ (where $N_{c}$ is the total number of the frustrated columns with nonzero $q_{x}^{-}$), we can finally rewrite the effective weak (dipolar) interaction as

$$
\sum_{x, x^{\prime}} q_{x}^{-} q_{x^{\prime}}^{-} U^{-}\left(x-x^{\prime}\right)=\sum_{s=1}^{N_{c}} U_{s} q_{s}^{-} q_{s+1}^{-}+U(0) \sum_{s=1}^{N_{c}}\left(q_{s}^{-}\right)^{2},
$$

where $q_{N_{c}+1} \equiv q_{1}$ for PBC/APBC and $q_{N_{c}+1} \equiv-q_{1}$ for RBC. The charges $q_{s}^{-}$take the values $\pm \frac{1}{2}$ and \pm 1 , and the $U_{s}$ are independent quenched random interaction constants. Since in the set of states that we consider the Coulomb energy is boundary condition independent, we will deduce the energy differences, $\Delta E_{\mathrm{AP}}^{(N)}$ and $\Delta E_{\mathrm{R}}^{(N)}(N \rightarrow \infty)$, from (4.4) and from the global spin wave term in (2.28). The large $N$ limit is selfunderstood in what follows.

\subsection{Antiperiodic boundary conditions}

The actual ground state minimises the second and third term in $\mathcal{H}_{\mathrm{P}}$ and $\mathcal{H}_{\mathrm{AP}}$, equation (2.28), within the space of degenerate ground states of the Coulomb energy, characterised in the preceding section. The second term, rewritten in equation (4.4), is the weak interaction between the $q_{x}^{-}$. In Appendix B we show that its lowest-lying excitation lies typically an energy amount $\sim J N^{-y_{c}}$ above the ground state, and determine the exponent $y_{c}=\log _{\frac{8}{3}}(3+2 \sqrt{2})=1.7972 \ldots$ The third term is

$$
\begin{array}{ll}
\frac{8 \pi^{2}}{N}\left(n+\frac{1}{2} \sum_{x} q_{(x, 1)}+\sum_{x} \frac{\pi_{(x, 1)}}{2 \pi}\right)^{2} & \text { in } \mathcal{H}_{\mathrm{P}}, \\
\frac{8 \pi^{2}}{N}\left(n+\frac{1}{2} \sum_{x} q_{(x, 1)}+\sum_{x} \frac{\pi_{(x, 1)}}{2 \pi}+\frac{1}{2}\right)^{2} & \text { in } \mathcal{H}_{\mathrm{AP}},
\end{array}
$$


where we have used the neutrality condition to write $\sum_{x} q_{x}^{-}=2 \sum_{x} q_{(x, 1)}$. The terms in (4.5), of order $N^{-1}$, have their origin in a global spin wave, i.e., of wave length $>N$, which helps the system to adjust to its boundary conditions when there is a rotational mismatch (cf. [8, 9).

One might wonder if it is always possible, by choosing $n$ properly, that the terms in (4.5) vanish in the ground state in $\mathcal{H}_{\mathrm{P}}$ and/or $\mathcal{H}_{\mathrm{AP}}$. As $n+\sum_{x=1}^{N} \frac{\pi_{(x, 1)}}{2 \pi} \in\left\{0, \pm \frac{1}{2}, \pm 1, \ldots\right\}$, this depends obviously on the number of nonzero charges $q_{(x, 1)}$. Hence we have two cases:

(i) the number of frustrated plaquettes $(x, 1)$ is even;

(ii) the number of frustrated plaquettes $(x, 1)$ is odd.

Correspondingly:

$$
\begin{array}{ll}
\frac{1}{2} \sum_{x=1}^{N} q_{(x, 1)} \in\left\{0, \pm \frac{1}{2}, \pm 1, \ldots\right\} & \text { case }(i), \\
\frac{1}{2} \sum_{x=1}^{N} q_{(x, 1)} \in\left\{ \pm \frac{1}{4}, \pm \frac{3}{4}, \ldots\right\} & \text { case }(i i) .
\end{array}
$$

We investigate these cases further.

(i) Even number of frustrated plaquettes $(x, 1)$ : Given the set of $\pi_{(x, 1)}$ and possibly reversing a sequence of dipoles as in Appendix B to get $\frac{1}{2} \sum_{x} q_{(x, 1)}+\sum_{x} \frac{\pi_{(x, 1)}}{2 \pi}$ integer for PBC and halfinteger for APBC or vice versa, the terms in (4.5) vanish for both Hamiltonians by a proper choice of $n$. As there is a difference of $\frac{1}{2}$ in the term in parentheses in (4.5), the ground states of $\mathcal{H}_{\mathrm{P}}$ and $\mathcal{H}_{\mathrm{AP}}$ differ by a reversal of a sequence of dipoles containing an odd number of $q_{(x, 1)} \neq 0$, i.e., a sequence of dipoles among which an odd number is slanted. Hence we obtain, reinserting $J$,

$$
\Delta E_{\mathrm{AP}}^{(N)} \sim \pm J N^{-y_{c}}, \quad \text { case }(i)
$$

where the sign indicates that either state, at PBC or APBC, has the lower ground state energy.

(ii) Odd number of frustrated plaquettes $(x, 1)$ : Here, from (4.6), the terms (4.5) in $\mathcal{H}_{\mathrm{P}}$ and $\mathcal{H}_{\mathrm{AP}}$ are always nonzero and the optimal value of $n$ will give an energy $J \frac{\pi^{2}}{4 N}$ irrespective of the directions of the dipoles. These will thus be determined by the weak interaction only and be the same for both boundary conditions. The energy difference is hence

$$
\Delta E_{\mathrm{AP}}^{(N)}=0, \quad \text { case }(i i),
$$

in this case.

\subsection{Reflecting boundary conditions}

The ground states at PBC and RBC minimise the second and the third term in $\mathcal{H}_{\mathrm{P}}$ and the second term in $\mathcal{H}_{\mathrm{R}}$, equation (2.28), within the set of states characterised in the preceding section. So, again, one has to distinguish between an even and odd number of $q_{(x, 1)} \neq 0$, when calculating the typical ground state energy difference. 
(i) Even number of frustrated plaquettes $(x, 1)$ : We saw in section 4.2 that the term for $\mathrm{PBC}$ in 4.5 ) vanishes in the ground state of $\mathcal{H}_{\mathrm{P}}$ and that, for half of the samples, one finds the ground state at PBC by minimising the weak (dipolar) interaction. If one changes to $\mathrm{RBC}$ for this half of the samples, while keeping the PBC ground state configuration, one will almost always be able to lower the energy [Note that the sign of the bond that passes column $N$, which is almost never the weakest one, has changed (!)]: one just has to reverse a sequence of dipoles starting at column $N$ in such a way that only one of the weakest bonds is broken. For the other half of the samples, the cancellation of the global spin wave term implies a reversal of a sequence of dipoles in the configuration after minimisation of the dipolar interaction. For these latter samples, when one changes to RBC, one has to reverse again the same sequence that was reversed to get the ground state at PBC. In both cases, this leads to

$$
\Delta E_{\mathrm{R}}^{(N)} \sim \pm J N^{-y_{c}}, \quad \text { case }(i)
$$

for the difference in ground state energies. The minus sign applies for the first half of the samples, the plus for the second half.

(ii) Odd number of frustrated plaquettes $(x, 1)$ : The global spin wave term never vanishes in PBC, so that

$$
\Delta E_{\mathrm{R}}^{(N)} \simeq \frac{\pi^{2}}{4} J N^{-1}, \quad \text { case }(i i),
$$

neglecting a possible contribution of order $J N^{-y_{c}}$.

\section{Conclusion}

We have studied the $X Y$ spin glass with $\pm J$ bonds on a tube lattice. This system has both a continuous (spin) and a discrete (chiral) symmetry, and hence two order parameters play a rôle. Our purpose was to determine the divergence, for $T \rightarrow 0$, of the chiral and the spin correlation lengths, via the finite size scaling of the ground state energy differences under different boundary conditions. In the presence of two symmetries, the usual single-symmetry relation between the finite size scaling exponents of the ground state energy difference and the correlation length has to be extended in a nontrivial way. Nevertheless, the spin correlation length exponent $y_{s}$ (see equation (4.1)) is given by the energy difference when one passes from periodic to antiperiodic boundary conditions, namely ${\overline{\left(\Delta E_{\mathrm{AP}}^{(N)}\right)^{2}}}^{\frac{1}{2}} \sim J N^{-y_{s}}$. New boundary conditions, reflecting ones, were introduced [5] to determine the chirality correlation length exponent $y_{c}$.

The difficulty in performing such an analysis on a general $N \times M$ lattice is that one does not know how to construct the ground states of the disordered systems. The tube lattice, of this work, however, just as the ladder lattice studied earlier [8], allows for a precise theoretical analysis of this relation. In contrast to the ladder lattice, the tube lattice still has long-range interactions between its chiralities, and is therefore closer to a two-dimensional system.

We first apply the well-known transformation [2, 9] of the $X Y$ spin glass into a Coulomb gas, a system of chiral variables (also called charges). The resulting effective Hamiltonian can be cast in the form (2.28) where it is the sum of three terms: 
(i) A one-dimensional Coulomb interaction, linearly increasing with distance, between charges $q_{1}^{+}$, $q_{2}^{+}, \ldots, q_{N}^{+}$; in (2.28), this term has been expressed as the volume sum of the energy density of the electric field $E_{x}$.

(ii) A "dipolar" interaction that decreases exponentially with distance between the $q_{1}^{-}, q_{2}^{-}, \ldots, q_{N}^{-}$.

(iii) The energy of a spin wave needed to match PBC or $A P B C$ (but absent under $R B C$ ), and whose wavenumber depends on the total electric dipole moment.

The third term disappears in the thermodynamic limit. Its relevance for a finite size scaling analysis was first pointed out by Fisher, Tokuyasu and Young [10]. Moreover, the three terms are, on the one hand, coupled by local constraints, that link the allowed values of $q_{x}^{+}$and $q_{x}^{-}$with the fixed values of the ferromagnetic or antiferromagnetic bonds $\pi_{i j}$ between the spins on the lattice, and, on the other hand, by a global constraint on the total charge (zero for PBC and APBC, and even or uneven for RBC). Taking these constraints into account, we identify the low-lying excitations of the three terms, respectively:

(i) Coulomb excitations that cost an energy of order $J$.

(ii) Chiral excitations, obtained by reversing a sequence of chiral variables, that cost an energy $J N^{-y_{c}}$ with $y_{c}=1.7972 \ldots$.

(iii) Global spin waves that cost an energy $\sim J N^{-1}$.

The $\pm J X Y$ spin glass on the ladder lattice [8] consists of both interactions (ii) and (iii). Due to the additional long-range interaction (i), the tube is closer to the two-dimensional model.

In spite of the number of interactions in competition, we were able to characterise and delimit the set of charge configurations, within which lies the ground state. In the configurations contained in this set, the charges take the values $\pm \frac{1}{2}$ on the frustrated plaquettes and zero on the others, and form a chain of dipoles.

We now give a summary of our results, and recall numerical results for comparison. When changing boundary conditions from $\mathrm{PBC}$ to $\mathrm{APBC}$, or RBC, it is the excitations (ii) and (iii) that give both energy differences, $\Delta E_{\mathrm{AP}}^{(N)}$ and $\Delta E_{\mathrm{R}}^{(N)}$. This implies the same conclusions as in [8]: Firstly, the ground state obtained with $\mathrm{P}$ boundary conditions can adjust to AP boundary conditions via a chiral excitation, so that

$$
{\overline{\left(\Delta E_{\mathrm{AP}}^{(N)}\right)^{2}}}^{\frac{1}{2}} \sim J N^{-y_{c}}, \quad y_{c}=1.7972 \ldots .
$$

The last equation contains no reference to spin waves and means that $y_{s}=y_{c}$. Secondly, passing from $\mathrm{P}$ to $\mathrm{R}$ boundary conditions releases a global spin wave (as was first observed by Kawamura and Tanemura [5] in $d=2$ ) in half of the samples, but does not do so in the other half.

In $d=2$, Kawamura and Tanemura performed a numerical analysis of the different ground state energies of the cosine $X Y$ model. They find, as $N \rightarrow \infty$,

$$
\begin{aligned}
& {\overline{\left(\Delta E_{\mathrm{AP}}^{(N)}\right)^{2}}}^{\frac{1}{2}} \quad \simeq a N^{-y_{s}}, \quad y_{s} \approx 0.84, \\
& {\overline{\left(\epsilon_{\mathrm{R}}^{(N)}-{\overline{\epsilon_{\mathrm{R}}^{(N)}}}^{2}\right.}}^{\frac{1}{2}} \simeq b N^{-y_{c}}+\mathcal{O}\left(N^{-y_{s}}\right), \quad y_{c} \lesssim 0.38 \text {, }
\end{aligned}
$$


where $a$ and $b$ are constants, and a new quantity, namely

$$
\epsilon_{\mathrm{R}}^{(N)} \equiv \Delta E_{\mathrm{R}}^{(N)}-\min \left(0, \Delta E_{\mathrm{AP}}^{(N)}\right)
$$

has been introduced. Thus, they get two distinct exponents $y_{s}$ and $y_{c}$, with $y_{s}>y_{c}$, and conclude that the chiralities order on a longer scale than the spin variables.

For the tube, upon collecting our results [equations (4.7),(4.8),(4.9) and (4.10)], we get

$$
\overline{\left(\epsilon_{\mathrm{R}}^{(N)}-{\overline{\epsilon_{\mathrm{R}}^{(N)}}}^{2}{ }^{\frac{1}{2}}\right.}=\frac{\pi^{2}}{8} J N^{-1}+\mathcal{O}\left(N^{-y_{c}}\right),
$$

i.e., the $\mathrm{R}$ boundary conditions probe a global spin wave term proportional to $N^{-1}$. If we now conjecture on the extrapolation of our results to $d=2$, then we expect for the quantities of equation (5.2) that $\Delta E_{\mathrm{AP}}$ would yield a chiral exponent $y_{c}$ as in (5.1) but with a smaller value (since $y_{c}$ should vanish at some, still higher, lower critical dimension); and that $\epsilon_{\mathrm{R}}$ would yield the spin wave exponent $d-2=0$. Instead, in contrast, Kawamura and Tanemura interpret their simulation according to (5.2). We expect that simulations on larger $2 d$ systems will confirm our scenario.

\section{Appendix A}

In this appendix, we prove that in the large $N$ limit the ground states of the system for the different boundary conditions possess the properties 1 to 3 announced in section III. In the calculations, we write the expression of the weak (dipolar) interaction in its form at PBC/APBC (equation (2.12)), with again $J=2$. The arguments are nevertheless readily rewritten for $\mathrm{RBC}$, including the appropriate factors of $\sigma\left(x, x^{\prime}\right)$ (equation (2.20)). Furthermore, we neglect the global spin wave term $\mathcal{O}\left(\frac{1}{N}\right)$ that

appears for PBC/APBC. Upon proper choice of $n$, this term contributes at most $\frac{\pi^{2}}{2 N}$ to the ground state energies at PBC/APBC, which is small for $N \rightarrow \infty$, in comparison to the other energies involved.

In preparation of the proofs of the ground state properties 1 to 3 , we show, in a first step, that

(i) in the ground state, the charges $q_{x}^{-}$take values $\left|q_{x}^{-}\right| \leqq \frac{3}{2}$,

and, using (i), in a second step, that

(ii) in the ground state, the charges $q_{x}^{-}$take values $\left|q_{x}^{-}\right| \leqq 1$. 


\section{Proofs of (i) and (ii):}

(i) In the ground state, the charges $q_{x}^{-}$take values such that $\left|q_{x}^{-}\right| \leqq \frac{3}{2}$.

Let us look at some state with charges $q_{x}^{-, 0}$ such that

$$
q \equiv \max \left\{\left|q_{x}^{-, 0}\right|\right\} \geqq 2 .
$$

Let $q_{x_{0}}^{-, 0}$ be a charge with $\left|q_{x_{0}}^{-, 0}\right|=q$. For reasons of charge reversal symmetry, we may take $q_{x_{0}}^{-, 0}$ positive without loss of generality.

Consider now the state in which $q_{x_{0}}^{-, 0}$ is changed into $q_{x_{0}}^{-, 0}-2$, while all other charges $q_{x}^{-, 0}$ and all charges $q_{x}^{+, 0}$ are kept unchanged. [Note that, by an appropriate change of $q_{(x, 1)}$ and $q_{(x, 2)}$, one can add an arbitrary multiple of 2 to some charge $q_{x}^{-}$without changing $q_{x}^{+}$(see (2.9)).] The difference in energy $\Delta E$ between the (final) state, with $q_{x_{0}}^{-, 0}$ changed, and the initial state can readily be calculated. As the charges $q_{x}^{+}$are unchanged, it comes from the difference in weak interaction energy only. For $q_{x_{0}}^{-, 0} \geqq 2$, one finds

$$
\begin{aligned}
\Delta E & =\frac{\sqrt{2}}{8} \pi^{2}\left[(q-2)^{2}-q^{2}\right]+\frac{\sqrt{2}}{8} \pi^{2}[q-(q-2)] 2 \sum_{s>0}\left(q_{x+s}^{-, 0}+q_{x-s}^{-, 0}\right)(3-2 \sqrt{2})^{s} \\
& =\frac{\sqrt{2}}{8} \pi^{2}\left[-4(q-1)+4 \sum_{s>0}\left(q_{x+s}^{-, 0}+q_{x-s}^{-, 0}\right)(3-2 \sqrt{2})^{s}\right] .
\end{aligned}
$$

We have

$$
\left|4 \sum_{s>0}\left(q_{x+s}^{-, 0}+q_{x-s}^{-, 0}\right)(3-2 \sqrt{2})^{s}\right| \leqq 8 q\left|\sum_{s>0}(3-2 \sqrt{2})^{s}\right|,
$$

and thus, summing the geometric series and using $q \geqq 2$,

$$
\Delta E \leqq \frac{\sqrt{2}}{8} \pi^{2}[-4(q-1)+4(\sqrt{2}-1) q]<0 .
$$

So the final state is lower in energy than the initial state. Hence a state, in which $q \geqq 2$, is not the ground state.

(ii) In the ground state, the charges $q_{x}^{-}$take values such that $\left|q_{x}^{-}\right| \leqq 1$.

Let us take some state with charges $q_{x}^{-, 0}=0, \pm \frac{1}{2}, \pm 1, \pm \frac{3}{2}$. Be $n$ the number of charges $\pm \frac{3}{2}$, with $n>0$. Suppose for the moment that $n<N$. There exists hence a sequence of charges $q_{x_{0}}^{-, 0}, q_{x_{0}+1}^{-, 0}, \cdots$, $q_{x_{0}+n^{\prime}-1}^{-, 0}= \pm \frac{3}{2}\left(1 \leqq n^{\prime} \leqq n\right)$ that is enclosed between charges $q_{x}^{-, 0}$ of absolute value $\leqq 1$ (i.e., $\left.\left|q_{x_{0}-1}^{-, 0}\right|,\left|q_{x_{0}+n^{\prime}}^{-, 0}\right| \leqq 1\right)$.

Consider now the state in which all charges $q_{x}^{-, 0}= \pm \frac{3}{2}$ in this sequence are replaced by $\mp \frac{1}{2}$, while all other charges $q_{x}^{-, 0}$ and all charges $q_{x}^{+, 0}$ are kept unchanged. As in (i), the difference in energy $\Delta E$ between the (final) state, with the changes, and the initial state is due to the difference in weak (dipolar) interaction energy only. The difference $\Delta E_{0}$ in weak interaction energy, coming from the self-interaction terms in the sum, is

$$
\Delta E_{0}=-2 \frac{\sqrt{2}}{8} \pi^{2} n^{\prime}
$$

the one from the nearest-neighbour interaction terms is of absolute value

$$
\left|\Delta E_{1}\right| \leqq 2 \frac{\sqrt{2}}{8} \pi^{2}(3-2 \sqrt{2})\left[\left(n^{\prime}-1\right) \frac{9}{4}+2 \cdot \frac{3}{2} \cdot 1\right]+2 \frac{\sqrt{2}}{8} \pi^{2}(3-2 \sqrt{2})\left[\left(n^{\prime}-1\right) \frac{1}{4}+2 \cdot \frac{1}{2} \cdot 1\right],
$$


while the energy difference of all other terms is of absolute value

$$
\left|\Delta E_{2}\right| \leqq 4 \frac{\sqrt{2}}{8} \pi^{2} \frac{(3-2 \sqrt{2})^{2}}{1-(3-2 \sqrt{2})} \frac{9}{4} n^{\prime}+4 \frac{\sqrt{2}}{8} \pi^{2} \frac{(3-2 \sqrt{2})^{2}}{1-(3-2 \sqrt{2})} \frac{3}{4} n^{\prime} .
$$

To obtain the last inequality, we have substituted for all charges $q_{x}^{-}$, but the ones in the sequence, the maximal possible absolute value $\frac{3}{2}$ and taken all terms in the sum to be negative in the initial state and positive in the final state [which is obviously an upper bound for the energy change, but impossible to realise]. The overall energy difference is thus

$$
\begin{aligned}
\Delta E & \leqq-2 \frac{\sqrt{2}}{8} \pi^{2} n^{\prime}+\frac{\sqrt{2}}{8} \pi^{2}(3-2 \sqrt{2})\left[5\left(n^{\prime}-1\right)+8\right]+12 \frac{\sqrt{2}}{8} \pi^{2} \frac{(3-2 \sqrt{2})^{2}}{1-(3-2 \sqrt{2})} n^{\prime} \\
& =-\frac{\sqrt{2}}{8} \pi^{2}\left[n^{\prime}(29-20 \sqrt{2})-9+6 \sqrt{2}\right]<0 .
\end{aligned}
$$

Again, the energy of the final state is lower than the energy of the initial state. If $n=N$, a similar reasoning (with $n^{\prime}=n=N$ ) leads to the same conclusion. This proves (ii).

\section{We are now prepared to show that the ground state has the properties 1 to 3 , announced in section III.}

1. In the ground state, the electric field satisfies $\left|E_{x}\right| \leqq \frac{1}{2}$. The constant background field takes values $\left|E_{0}\right| \leqq \frac{1}{2}$.

We first note that the electric field is constrained to be of absolute value $\leqq 1$ for the ground state configuration. This can be seen as follows. From (2.28), we see that minimisation of the Coulomb interaction part of the Hamiltonians means minimising the mean square value of the local electric field $E_{x}$. This leads for all Hamiltonians to

$$
E_{0}=\left\{\begin{aligned}
0, & \text { if } \frac{\pi_{N}^{12}+\pi_{N}^{21}}{\pi} \bmod 2=0 \\
\pm \frac{1}{2}, & \text { if } \frac{\pi_{N}^{12}+\pi_{N}^{21}}{\pi} \bmod 2=1
\end{aligned}\right.
$$

for the constant background field $E_{0}$. The electric field, $E_{x}$, should be locally optimal, that is stay as close to 0 as possible. For any given state with given sets of charges $\left\{q_{x}^{+}\right\}$and $\left\{q_{x}^{-}\right\}$, one can go successively through the system, from $x=1$ to $x=N$, changing, whenever necessary, the charges $q_{x}^{+}$ in such a way that the value of the electric field is bounded in absolute value by 1 in the final state: At every column with no frustrated or two frustrated plaquettes, the change in electric field can be bounded to be $0, \pm 1$ by an appropriate change of $q_{x}^{+}$, if necessary, and to be $\pm \frac{1}{2}$ on every column with exactly one frustrated plaquette. [By the definition of $q_{x}^{+}$and $q_{x}^{-}$, equation (2.9), one can add an arbitrary multiple of 2 to some charge $q_{x}^{+}$without changing $q_{x}^{-}$, by an appropriate change of $q_{(x, 1)}$ and

$q_{(x, 2)}$.] Whenever one encounters a column $x_{0}$, during the above procedure, where the electric field jumps to a value $\left|E_{x_{0}}\right| \geqq \frac{3}{2}$, one changes $q_{x_{0}}^{+}$by the appropriate amount, as well as the next nonzero charge $q_{x}^{+}$, say at $x_{0}^{\prime}$, by the opposite amount (to conserve charge neutrality). By the definition of the electric field, cf. equation (2.22), every time one changes the charges at $x_{0}$ and $x_{0}^{\prime}$ only, the electric field remains unchanged on columns $x<x_{0}$ and $x \geqq x_{0}^{\prime}$. So in the end, while keeping the set of charges $\left\{q_{x}^{-}\right\}$fixed, the value of the electric field is bounded in absolute value by 1 . In particular, the final state is lower in energy than the initial state. 
Let us now take a charge configuration of the system (with charges $q_{x}^{+, 0}, q_{x}^{-, 0}$ ) such that the electric field jumps, say at column $x_{0}$, to a value $\left|E_{x_{0}}\right|=1$, and stays at this value until column $x_{0}^{\prime}\left(>x_{0}\right)$, where one finds hence the next nonzero charge $q_{x}^{+, 0}$. Consider the state in which $q_{x_{0}}^{+, 0}$ is changed by an amount of 1 to some value $\left|E_{x_{0}}^{\prime}\right| \leqq \frac{1}{2}$ and $q_{x_{0}^{\prime}}^{+, 0}$ by the opposite amount (to conserve charge neutrality), while keeping the charges $q_{x}^{+, 0}$ (and $q_{x}^{-, 0}$ ) on all other columns fixed. We observe that, from (2.22), the electric field is unchanged for $x<x_{0}$ and $x \geqq x_{0}^{\prime}$ and that by the definition of $q_{x}^{+}$and $q_{x}^{-}$, the charges $q_{x_{0}}^{-, 0}$ and $q_{x_{0}^{\prime}}^{-, 0}$ changed. Anyhow, from (ii), the absolute value of the charges $q_{x}^{-}$can be taken to be bounded by 1 in both states, as otherwise the energy of the state can still be lowered. The difference $\Delta E^{F}$ in strong interaction energy between the final state and the initial state is

$$
\Delta E^{F} \leqq-2 \pi^{2} \frac{3}{4}\left|x_{0}-x_{0}^{\prime}\right|,
$$

while the difference $\Delta E^{f}$ in weak interaction energy is bounded by

$$
\begin{aligned}
\left|\Delta E^{f}\right| \leqq & 2 \frac{\sqrt{2}}{8} \pi^{2}\left[1+2\left|\sum_{s>0} q_{x_{0}}^{-, 0}\left(q_{x_{0}+s}^{-, 0}+q_{x_{0}-s}^{-, 0}\right)(3-2 \sqrt{2})^{s}\right|\right] \\
& +2 \frac{\sqrt{2}}{8} \pi^{2}\left[1+2\left|\sum_{s>0} q_{x_{0}^{\prime}}^{-, 0}\left(q_{x_{0}^{\prime}+s}^{-, 0}+q_{x_{0}^{\prime}-s}^{-, 0}\right)(3-2 \sqrt{2})^{s}\right|\right] .
\end{aligned}
$$

[The factors 2 in the last inequality stem from the fact that both the energy of the initial state and the one of the final state enter in the difference.] Hence we get for the total energy difference

$$
\Delta E \leqq-2 \pi^{2} \frac{3}{4}+4 \frac{\sqrt{2}}{8} \pi^{2}[1+2(\sqrt{2}-1)]<-\frac{\sqrt{2}-1}{2} \pi^{2},
$$

i.e., the energy of the final state is lower than the one of the initial state. Thus $\left|E_{x}\right| \leqq \frac{1}{2}$ for the ground state configuration.

2. In the ground state, the charges $q_{(x, 1)}, q_{(x, 2)}$ take the values $0, \pm \frac{1}{2}$.

From (ii) and 1, the absolute values of the charges on the plaquettes in the ground state are bounded by 1 . In the case that $E_{x-1}=0$ in the ground state, it is easy to see from (ii) and 1 that the charges $q_{(x, 1)}, q_{(x, 2)}$ on the plaquettes of column $x$ are of value $0, \pm \frac{1}{2}$.

Let us thus consider the case $\left|E_{x-1}\right|=\frac{1}{2}$. If there is at least one frustrated plaquette on column $x$, it is again obvious from (ii) and 1 that $q_{(x, 1)}$ and $q_{(x, 2)}$ are of absolute value $\leqq \frac{1}{2}$. Suppose now that there are two nonfrustrated plaquettes on a certain column $x_{0}$. From what we have stated at the beginning of this paragraph, it could be that there is a charge \pm 1 on one of the plaquettes. Let us furthermore suppose that there is a sequence of columns $x_{0}, x_{0}+1, \cdots, x_{0}+n-1$ that carry charges $q_{x}^{-}$of absolute value 1.

If the number $n$ of charges $q_{x}^{-}$in the sequence is even, consider the state in which all the charges $q_{x_{0}}^{-}, q_{x_{0}+1}^{-}, \cdots, q_{x_{0}+n-1}^{-}$are changed to 0 [this is possible as it conserves charge neutrality, as one convinces oneself with the help of 1]. The absolute value of the electric field is the same in both the initial and the final state, so that the difference in energy is again due to the weak interaction only:

$$
\Delta E \leqq-\frac{\sqrt{2}}{8} \pi^{2} n+4 \frac{\sqrt{2}}{8} \pi^{2} n \frac{3-2 \sqrt{2}}{1-(3-2 \sqrt{2})}=\frac{\sqrt{2}}{8} \pi^{2} n[2 \sqrt{2}-3]<0
$$

and the energy of the final state is lower than the one of the initial state. 
In the case that $n$ is odd, one can take $n=1$ and $\left|q_{x-1}^{-}\right|,\left|q_{x+1}^{-}\right| \leqq \frac{1}{2}$ without loss of generality, in view of the preceding paragraph. Suppose first that one of the charges $q_{x-1}^{-}, q_{x+1}^{-}$is of absolute value $\frac{1}{2}$ in the ground state, say $q_{x+1}^{-}$and say $\left|q_{(x+1,1)}\right|=\frac{1}{2}$. Let us compare the energy of this state with the one in which $q_{x}^{-}$is changed to 0 and $q_{(x+1,1)}$ replaced by $-q_{(x+1,1)}$ [note that $q_{x}^{-}+q_{(x+1,1)}=-q_{(x+1,1)}$ from 1 , so that the absolute value of the electric field remains unchanged and charge neutrality is conserved]. Using (ii), the difference in energy is

$$
\begin{aligned}
\Delta E \leqq \frac{\sqrt{2}}{8} \pi^{2}[-1 & +2\left(\left|q_{x-1}^{-, 0} q_{x}^{-, 0}\right|+\left|q_{x}^{-, 0} q_{x+1}^{-, 0}\right|+\left|q_{x+1}^{-, 0} q_{x+2}^{-, 0}\right|\right)(3-2 \sqrt{2}) \\
& +2\left|-q_{x+1}^{-, 0} q_{x+2}^{-, 0}\right|(3-2 \sqrt{2})+2 \sum_{s>1}\left|q_{x}^{-, 0}\right|\left|q_{x-s}^{-, 0}+q_{x+s}^{-, 0}\right|(3-2 \sqrt{2})^{s} \\
& \left.+2 \cdot 2 \sum_{s>1}\left|q_{x+1}^{-, 0}\right|\left|q_{x+1-s}^{-, 0}+q_{x+1+s}^{-, 0}\right|(3-2 \sqrt{2})^{s}\right] \\
\leqq & \frac{\sqrt{2}}{8} \pi^{2}\left[-1+8(3-2 \sqrt{2}) \frac{1}{2}+8 \frac{(3-2 \sqrt{2})^{2}}{2 \sqrt{2}-2}\right]=\frac{\sqrt{2}}{8} \pi^{2}(-17+12 \sqrt{2})<0 .
\end{aligned}
$$

Again the final state is lower in energy than the initial state. Secondly, if $q_{x-1}^{-}=q_{x+1}^{-}=0$, there are again two possibilities. Either there are only nonfrustrated plaquettes on the columns $x-1$ and $x+1$, or, on at least one of them, both plaquettes are frustrated, say at $x+1$. In the last case, one has $q_{x}^{+}=-2 q_{(x+1,1)}=-2 q_{(x+1,2)}$ from 1 ; hence changing $q_{x}^{-}$to 0 and $q_{(x+1,1)}, q_{(x+1,2)}$ to $-q_{(x+1,1)},-q_{(x+1,2)}$ conserves charge neutrality, the absolute value of the electric field and does not change $q_{x-1}^{-}=q_{x+1}^{-}=0$. Using (ii), the energy difference between the final and the initial state is then

$$
\begin{aligned}
\Delta E & \leqq \frac{\sqrt{2}}{8} \pi^{2}\left[-1+2 \sum_{s>1}\left|q_{x}^{-, 0} \| q_{x-s}^{-, 0}+q_{x+s}^{-, 0}\right|(3-2 \sqrt{2})^{s}\right] \\
& \leqq \frac{\sqrt{2}}{8} \pi^{2}\left[-1+4 \frac{(3-2 \sqrt{2})^{2}}{2 \sqrt{2}-2}\right]=\frac{\sqrt{2}}{8} \pi^{2}(-15+10 \sqrt{2})<0 .
\end{aligned}
$$

In the case that $q_{(x-1,1)}=q_{(x-1,2)}=q_{(x+1,1)}=q_{(x+1,2)}=0$, there is a column $x-s$ or $x+s$ such that $q_{(x-\sigma, 1)}=q_{(x-\sigma, 2)}=q_{(x+\sigma, 1)}=q_{(x+\sigma, 2)}=0$ for all $1<\sigma<s$ and that one of the charges $q_{(x-s, 1)}, q_{(x-s, 2)}, q_{(x+s, 1)}, q_{(x+s, 2)}$ is nonzero. Considerations analoguous to the ones earlier in this paragraph lead again to the conclusion that a state in which there is a nonzero charge on a column with two nonfrustrated plaquettes is not the ground state.

So in the ground state, the charges $q_{(x, 1)}, q_{(x, 2)}$ take the values $0, \pm \frac{1}{2}$ only.

3. In the ground state, the charges $q_{(x, 1)}$ and $q_{(x, 2)}$ are equal on doubly frustrated columns, if and only if $\left|E_{x-1}\right|=\frac{1}{2}$.

Following exactly the same lines as in 2 , one shows that a state in which $q_{\left(x_{0}, 1\right)}=-q_{\left(x_{0}, 2\right)}$, i.e., $\left|q_{x_{0}}^{-}\right|=1$, on some doubly frustrated column $x_{0}$, while $\left|E_{x_{0}-1}\right|=\frac{1}{2}$, is higher in energy than the one in which $q_{\left(x_{0}, 1\right)}=q_{\left(x_{0}, 2\right)}$, i.e., $\left|q_{x_{0}}^{-}=0\right|$ [the appropriate change of some other charge, as in 2 , to conserve charge neutrality, is tacitly understood]. It is evident from 1 that, in the ground state, $q_{\left(x_{0}, 1\right)}=-q_{\left(x_{0}, 2\right)}$ on some doubly frustrated column $x_{0}$, if $E_{x_{0}-1}=0$. 


\section{Appendix B}

We calculate the typical energy change in a tube of length $N$, in the limit $N \rightarrow \infty$, when a sequence of dipoles is reversed to adjust to APBC or RBC from PBC, as in section IV. This amount of energy is related to the typical length of the longest interval that contains no nonzero charges $q_{x}^{-}$between two such (nonzero) charges. The reversals that are effectuated in section IV do not necessarily involve the longest of these intervals because of the constraints: one must not brake up a dipole, or in certain cases has to reverse not just any sequence, but one that contains an odd number of slanted dipoles. Nevertheless, these reversals will still typically involve intervals that are of the same order as the longest interval.

Since each plaquette is frustrated with probability $\frac{1}{2}$ independently of the others, a tube of length $N$ will typically contain $\frac{N}{4}$ nonfrustrated columns, $\frac{N}{2}$ with one frustrated plaquette, and $\frac{N}{4}$ where both plaquettes are frustrated. From property 3 of the ground state configuration, half of the doubly frustrated columns will typically contain charges that belong to the same dipole and the other half charges that belong to two different dipoles. This is due to the fact that at a given column $E_{x}=0$ or $E_{x}= \pm \frac{1}{2}$ (i.e., $q_{x}^{+}$integer or half-integer) with equal probability; in the first case, one has $q_{x}^{-}= \pm 1$, and in the other, $q_{x}^{-}=0$. So, there are more charges $q_{x}^{-}=0$ than there are nonfrustrated columns. Since it is typically half of the doubly frustrated columns that give $q_{x}^{-}=0$, we see that, again typically, $\frac{5}{8} N$ of the columns carry a nonzero charge $q_{x}^{-}$, while $\frac{3}{8} N$ of the columns are neutral in $q_{x}^{-}$. So the number of intervals between two nonzero charges $q_{x}^{-}$, that contain no other such (nonzero) charge, is $\frac{5}{8} N$. The probability $p(\ell)$ for the two subsequent nonvanishing charges $q_{x}^{-}$to be at a distance $\ell$ (i.e., to be separated by an interval of $\ell-1$ columns containing no nonzero charge $q_{x}^{-}$) is

$$
p(\ell)=\left(\frac{3}{8}\right)^{\ell-1} \frac{5}{8}, \quad \ell=1,2, \ldots
$$

Let $P_{N}(m)$ be the probability distribution for the length $m$ of the longest one of these distances. Obviously, $P_{N}(m)$ equals the probability that all $\frac{5}{8} N$ intervals have length $\ell \leqq m$, minus the probability that they all have length $\ell \leqq m-1$; explicitly

$$
P_{N}(m)=\left[\sum_{\ell=1}^{m} p(\ell)\right]^{\frac{5}{8} N}-\left[\sum_{\ell=1}^{m-1} p(\ell)\right]^{\frac{5}{8} N}=\left[1-\left(\frac{3}{8}\right)^{m}\right]^{\frac{5}{8} N}-\left[1-\left(\frac{3}{8}\right)^{m-1}\right]^{\frac{5}{8} N} .
$$

When $N$ is large, $P_{N}(m)$ will be peaked around some large value of $m$. Its scaling form can be obtained if one transforms from $m$ to $m^{\prime}$ according to

$$
m=\gamma \log N+m^{\prime}
$$

with $\gamma$ to be determined. Indeed, upon using $(\overline{B .3})$ and $(\overline{B .2})$, one finds

$$
P_{N}(m) \stackrel{N \rightarrow \infty}{\simeq} x\left(1-x^{\frac{5}{3}}\right), \quad \text { with } \quad x=e^{-\frac{5}{8}\left(\frac{3}{8}\right)^{m} N} .
$$

This shows that $P_{N}(m)$ is effectively nonzero only for argument values

$$
m=\log _{\frac{8}{3}} N+\mathcal{O}(1),
$$


and that the appropriate scaling limit reads

$$
\begin{aligned}
& N \rightarrow \infty \text { finite, fixed }, \\
& m^{\prime} \\
& \text { and } \gamma=\frac{1}{\log \frac{8}{3}} .
\end{aligned}
$$

Having thus obtained the typical length

$$
m=\frac{\log N}{\log \frac{8}{3}}
$$

of the longest interval, we are able to determine the typical energy change of the mentioned dipole reversals. From the effective weak interaction (4.4) we deduce that the typical energy change due to such a reversal is of order $c U(m)$, where

$$
U(m)=(3-2 \sqrt{2})^{m}
$$

is the energy change when breaking up a bond at distance $m$ and the constant $c$ is of order unity. The exponent $y_{c}$ is then obtained from the defining equation

$$
c U(0) N^{-y_{c}}=c U(m)
$$

which gives

$$
y_{c}=\log _{\frac{8}{3}}(3+2 \sqrt{2})=1.7972 \ldots
$$

for the exponent of the chirality-chirality correlation length.

\section{References}

[1] J. Villain, J. Phys. (Paris) 36 (1975) 582.

[2] J. Villain, J. Phys. C 10 (1977) 1717; J. Phys. C 10 (1977) 4793.

[3] J. Villain, J. Phys. C 11 (1977) 745.

[4] M. Schwartz and A.P. Young, Europhys. Lett. 15 (1991) 209; Y. Ozeki and H. Nishimori, Phys. Rev. B 46 (1992) 2879.

[5] H. Kawamura and M. Tanemura, Phys. Rev. B 36 (1987) 7177; J. Phys. Soc. Jpn. 60 (1991) 608.

[6] P. Ray and M.A. Moore, Phys. Rev. B 45 (1992) 5361.

[7] H. Kawamura, J. Phys. Soc. Jpn. 64 (1995) 26.

[8] M. Ney-Nifle, H.J. Hilhorst, and M.A. Moore, Phys. Rev. B 48 (1993) 10254.

[9] M. Ney-Nifle and H.J. Hilhorst, Phys. Rev. B, $1^{\text {st }}$ April 1995.

[10] M.P.A. Fisher, T.A. Tokuyasu, and A.P. Young, Phys. Rev. Lett. 66 (1991) 2931. 